\title{
Susceptibility of pentylenetetrazole-induced seizures in mice with Cereblon gene knockout
}

\author{
Seung-Je Jeon ${ }^{1, \#}$, Jinsil Ham ${ }^{2, \#}$, Chul-Seung Park ${ }^{1}$ E Boreom Lee ${ }^{2, *}$ \\ ${ }^{1}$ School of Life Sciences and Integrated Institute of Biomedical Research (IIBR), Gwangju Institute of Science and Technology (GIST), \\ Gwangju 61005, ${ }^{2}$ Department of Biomedical Science and Engineering (BMSE), Institute of Integrated Technology (IIT), Gwangju Institute \\ of Science and Technology (GIST), Gwangju 61005, Korea
}

Epilepsy is a neurological disorder characterized by unpredictable seizures, which are bursts of electrical activity that temporarily affect the brain. Cereblon (CRBN), a DCAFs (DDB1 and CUL4-associated factors), is a well-established protein associated with human mental retardation. Being a substrate receptor of the cullin-RING E3 ubiquitin ligase (CRL) 4 complex, CRBN mediates ubiquitination of several substrates and conducts multiple biological processes. In the central nervous system, the largeconductance $\mathrm{Ca}^{2+}$-activated $\mathrm{K}^{+}\left(\mathrm{BK}_{\mathrm{Ca}}\right)$ channel, which is the substrate of CRBN, is an important regulator of epilepsy. Despite the functional role and importance of CRBN in the brain, direct injection of pentylenetetrazole (PTZ) to induce seizures in CRBN knock-out mice has not been challenged. In this study, we investigated the effect of PTZ in CRBN knock-out mice. Here, we demonstrate that, compared with WT mice, CRBN knock-out mice do not show the intensification of seizures by PTZ induction. Moreover, electroencephalography recordings were also performed in the brains of both WT and CRBN knockout mice to identify the absence of significant differences in the pattem of seizure activities. Consistently, immunoblot analysis for validating the protein level of the CRL4 complex containing CRBN (CRL4 ${ }^{\mathrm{Crbn}}$ ) in the mouse brain was carried out. Taken together, we found that the deficiency of CRBN does not affect PTZ-induced seizure. [BMB Reports 2020; 53(9): 484489]

\section{INTRODUCTION}

Epilepsy is a serious brain disorder characterized by spontaneous

*Corresponding author. Tel: +82-62-715-3272; Fax: +82-62-715-3244; E-mail: leebr@gist.ac.kr

${ }^{\#}$ These authors contributed equally to this work.

https://doi.org/10.5483/BMBRep.2020.53.9.119

Received 4 June 2020, Revised 25 June 2020, Accepted 11 August 2020

Keywords: $\mathrm{BK}_{\mathrm{Ca}}$ channel, CRBN, Electroencephalography, Pentylenetetrazole, Seizure and recurrent seizures (1). Seizures may result from intense neuronal discharges generated by the synchronous activity of a large number of neurons in different brain regions, which result from an imbalance between excitatory and inhibitory neurotransmission (2). The large-conductance $\mathrm{Ca}^{2+}$-activated $\mathrm{K}^{+}$ $\left(\mathrm{BK}_{\mathrm{Ca}}\right)$ channel is extensively distributed in the central nervous system (CNS) and is considered as vital regulator for the pathogenesis of epilepsy (3). Pentylenetetrazole (PTZ) is a well-known noncompetitive $\mathrm{GABA}_{\mathrm{A}}$ receptor antagonist (4). A single injection of PTZ is widely used to induce seizures. In in vivo studies, the seizure activity induced by PTZ is classified into four stages: stage 1 is defined as the resting position, stage 2 is partial clonus, stages 3 and 4 are fully-induced whole body clonus and even death (5-7).

Cereblon (CRBN), initially identified as a target protein of mild autosomal recessive non-syndromic mental retardation (ARNSMR) (8), was subsequently revealed to regulate several different cellular functions. Being a substrate receptor of the cullin-RING E3 ubiquitin ligase (CRL) 4 complex, CRBN induces teratogenicity as the primary target for thalidomide (9) and renders anti-myeloma activity of immunomodulatory drugs (IMiDs) (10). In addition, CRBN directly interacts with the catalytic subunit of the AMP-activated protein kinase (AMPK) to negatively regulate its activity (11). The CRL4 complex containing CRBN $\left(\mathrm{CRL} 4^{\mathrm{Crbn}}\right)$ directly mediates the glutamine-induced degradation of glutamine synthetase (GS) (12), which is associated with diseases including Alzheimer's disease (13) and cancer (14). CRBN is also known to interact with various channel proteins such as voltage-gated chloride channel-2 (CIC-2) (15), which it degrades to control muscle excitability (16). CRBN also contributes to regulation of CD4 + T-cell activation via epigenetic regulation of Kv1.3 expression (17). The scope of research and subsequent reporting on the CRBN protein is gradually increasing.

CRBN is widely expressed in the post-mitotic period in most regions of the rodent brain, including the hippocampus, cerebral cortex, and cerebellum (18). The CRBN knockout (Crbn-KO) mouse generated by our research group (19) showed that altered activity of the $\mathrm{BK}_{\mathrm{Ca}}$ channel causes synaptic and cognitive dysfunction, which can be recovered with a BK $\mathrm{Ca}_{\mathrm{a}}$ channel blocker. Despite altered synaptic function, these mice show minor but significant differences in terms of learning and memory 
(20). CRBN was initially characterized as a regulator of $\mathrm{G}$ protein signaling (RGS) motif-containing novel protein interacting with the $\mathrm{BK}_{\mathrm{Ca}}$ channel in the rat brain (18). CRBN was then found to play an important role in the assembly and surface expression of functional $\mathrm{BK}_{\mathrm{Ca}}$ channels without altering its total protein amount through direct interaction with the cytosolic C-terminus of the alpha subunit. It has been suggested that the genetic ablation of DNA damage-binding protein 1 (DDB1) in post-mitotic neurons of the hippocampus and cerebral cortex increases susceptibility to PTZ-induced seizures (7). Furthermore, the role of the $\mathrm{K}^{+}$channel in epileptogenesis has been considered important for many years.

Despite the significant role of CBRN in brain function, as described above, it has never been elucidated whether Crbn$\mathrm{KO}$ mice are more sensitive to the induction of seizures by PTZ injection. In this study, we investigated the susceptibility to PTZ-induced seizure in Crbn-KO mice compared to that of wild-type controls.

\section{RESULTS}

\section{Deletion of Crbn had no obvious effects on susceptibility to PTZ-induced seizures}

PTZ is a well-known chemical used for experimentally-induced seizures; we assessed its efficacy and resulting seizures in a dose-dependent manner in Crbn-KO mice, previously generated by our research group (19). Several concentrations of PTZ (20200 mpk) had been used for susceptibility testing, and a wellestablished seizure stage scoring method was used for WT and Crbn-KO mice. Following 20 mpk PTZ, both WT and Crbn-KO mice exhibited seizures classified as stage 1 and stage 2 (Fig. 1A); mice were in a resting position with their abdomens completely touching the base of the cage. In some mice, we observed partial clonus throughout the body of the mice, including the head (Supplementary Video 1). Meanwhile, following a high dosage of PTZ (80 mpk), both WT and Crbn-KO mice exhibited severe seizures classified in stages 3 and 4 (Fig. 1B). These mice showed maximal clonus, seizures, and even death
(Supplementary Video 2). Moreover, following a higher dosage of PTZ (more than $120 \mathrm{mpk}$ ), both WT and Crbn-KO mice were inevitably led to death after maximal seizures (Supplementary Video 3). To summarize, the results showed that there were no significant differences between the WT and Crbn-KO mice in terms of the seizure stages and behaviors.

In a previous study, Ddb1-KO mice, lacking a core component of the CUL4 E3 ligase and a CRBN-binding partner, were reported to show relatively high sensitivity to PTZ injection (19). However, EEG recordings have never been performed using the Crbn-KO mouse. To visualize actual seizure activities, EEG were acquired after injection of PTZ into WT and Crbn$\mathrm{KO}$ mice. The signals were recorded from the motor cortex and hippocampus (Fig. 2B) using a couple of optimized devices, such as electrodes and connectors. Following $80 \mathrm{mpk}$ PTZ, the pattern of seizure activities recorded by EEG was similar in both groups (Fig. 2A). These observations are in line with the scoring of the seizure stages. Taken together, CRBN deficiency does not affect susceptibility to PTZ-induced seizures.
A

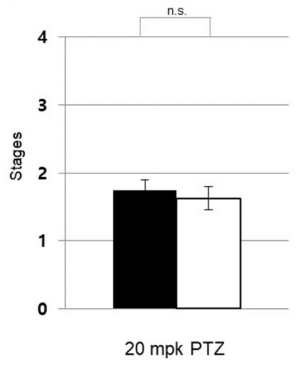

B

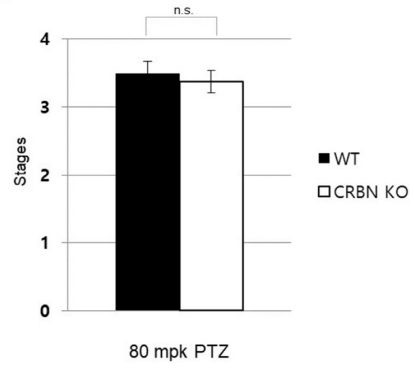

Fig. 1. PTZ-induced seizure stages. Scoring of seizure stages after (A) $20 \mathrm{mpk}$ and (B) $80 \mathrm{mpk}$ of PTZ. WT and Crbn-KO mice (C57BV 6) background, $\mathrm{n}=8$ for each group) were injected i.p. and recorded on video. Error bars indicate SEM and P-values are indicated.
A

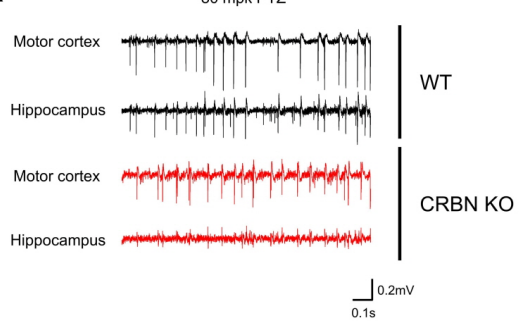

B

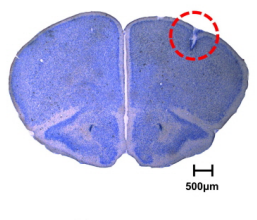

Motor cortex

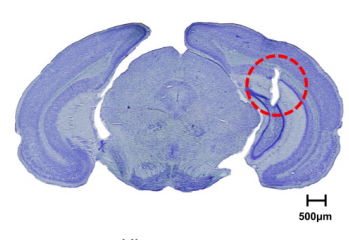

Hippocampus

Fig. 2. EEG recording of PTZ-induced seizure. (A) EEG signal was recorded from the motor cortex and hippocampus of WT and Crbn-KO mice. Both genotypes showed similar seizure patterns on EEG after 80 mpk PTZ (black: WT, red: Crbn-KO). EEG recordings confirmed that the degree of seizure activity across genotypes was not significantly different. (B) To verify that EEG signals were measured from the correct sites, brain slices were obtained. As shown above, implanted depth electrodes reached the motor cortex and hippocampus perfectly. 


\section{Crbn-KO in the mouse brain does not change the protein expression of E3-ubiquitin ligase machinery}

One of the most extensively studied functions of CRBN is its role as a substrate receptor for the CRL4 E3 ligase, which recognizes specific targets for their ubiquitination and degradation (21). CRBN forms an E3 ligase with DDB1, CUL4A, and regulator of cullins-1 (ROC1) to ubiquitinate many endogenous substrates. Since it is essential to check the protein level of E3 ligase machinery, Western blotting analysis was performed to analyze expression of DDB1 and CUL4 in the brain (cerebral cortex) of Crbn-KO mice (Fig. 3A). The kidney was used as a comparative tissue (Fig. 3F). The protein bands of CRBN in WT mice were obtained using a CRBN-specific polyclonal antibody with the exact molecular size $(53 \mathrm{kDa})$, and deficiency of CRBN in the brain and kidney of Crbn-KO mice were validated simultaneously. Overall, no statistical differences were found in the protein expression of CUL4 and DDB1, the major components of CRL4 ${ }^{\text {rrbn }}$-machinery, in the brain (Fig. 3B-D), and in the kidney (Fig. 3G-I); thus, confirming that despite CRBN deletion, the protein levels of DDB1 and CUL4 in the brain were not significantly changed. The protein level of $\mathrm{BK}_{\mathrm{Ca}}$ channel was simultaneously confirmed in each tissue of Crbn$\mathrm{KO}$ mice. We found that the protein expression of $\mathrm{BK}_{\mathrm{Ca}}$ channel was also unaffected by the deletion of CRBN and there was no change in its expression (Fig. 3E and 3J).

\section{DISCUSSION}

In this study, we investigated the effects of CRBN ablation on the electrical activity in the mouse brain using EEG recordings. We found that the lack of CRBN does not affect PTZ-induced seizures. However, several questions remain to be answered. First, we need to consider why our data showing that Crbn-KO mice do not show increased susceptibility to PTZ differs from that reported in Ddb1-KO mice, which shows vulnerability (19) despite relatively low concentrations of PTZ. In fact, CRBN and DDB1 are widely expressed throughout the rodent brain, including the hippocampus, cerebellum, and cerebral cortex (22). The aforementioned results showed that there was no difference in the expression of both proteins in the cerebral cortex of Crbn-KO mice. This indirectly implies that the ablation of DDB1 in mice might have a larger repercussion effect in the case of seizures. One plausible interpretation is that the complete disparity between the two knockouts effects from the functions of each respective protein. It stands to reason that the knockout effect of either DDB1 or CRBN in the CRLs is obviously different. CRBN is one of the diverse substrate receptors, called DCAFs. CUL4 ${ }^{\text {Crbn }}$ is one of the CRL E3-ubiquitin ligases, which encompass the largest E3 ligase family in eukaryotes and ubiquitinate a wide range of substrates that are responsible for numerous cellular processes (23). There are seven cullins (CUL1, 2, 3, 4A, 4B, 5, and 7) in vertebrates, and each cullin acts as a scaffold to interact with specific adapters, including DDB1, and diverse substrate receptors, including
A

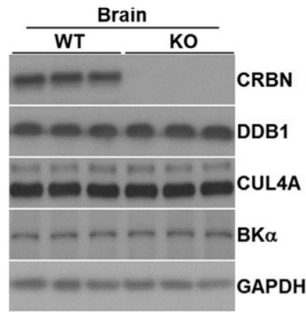

$\mathrm{F}$

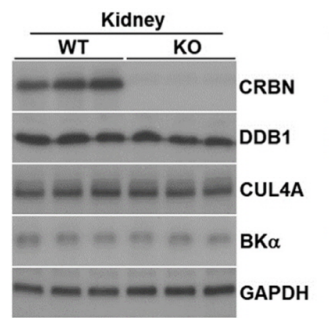

B

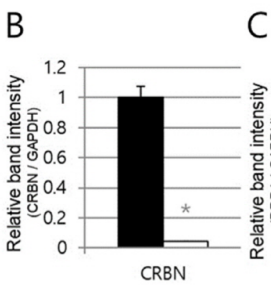

C

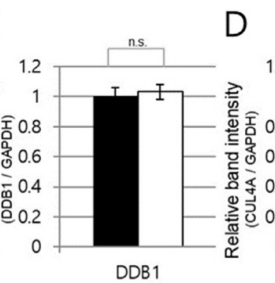

D

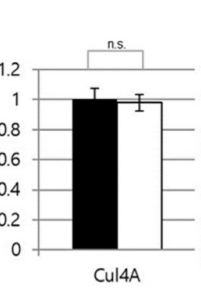

$\mathrm{E}$

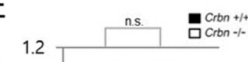

1.2
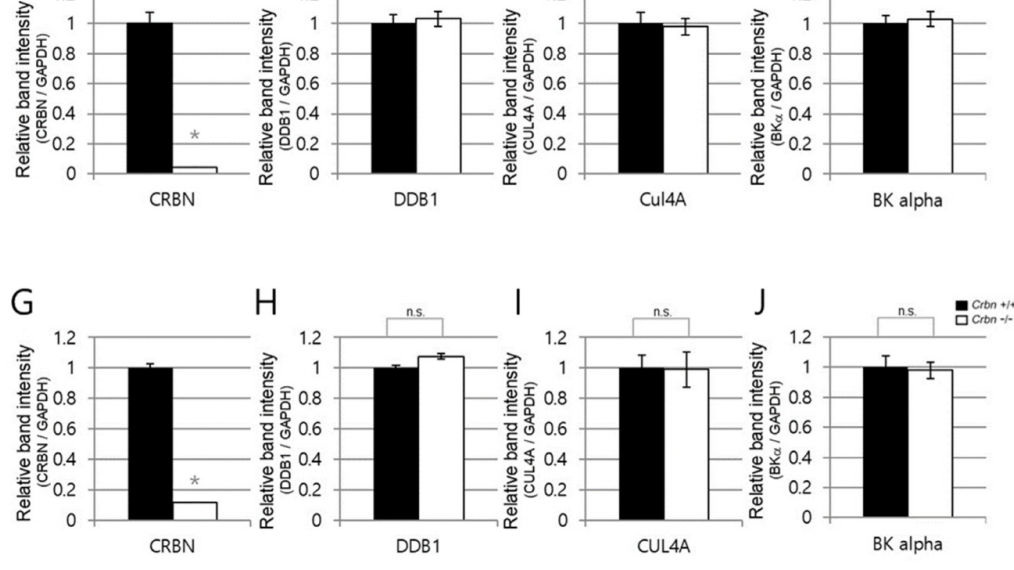

Fig. 3. Immunoblot analysis confirming the protein expression of components of the CRL4 ${ }^{\text {Crbn }}$ complex following the loss of CRBN. (A) and $(F)$ Western blot analysis confirming that loss of CRBN expression does not affect the protein levels of CUL4A, DDB1, and BKC channel in the brain (cerebral cortex) and kidney of the Crbn-KO mice. GAPDH was used as the loading control ( $\mathrm{n}=4$ for each group). (B-E) Relative protein band intensities of DDB1, CUL4A, and BK $\mathrm{Ca}$ channel of the blots in (A) measured by densitometer. (G-J) Relative protein band intensities of DDB1, CUL4A, and $\mathrm{BK}_{\mathrm{Ca}}$ channel determined by densitometric analysis of the blots in (F). The asterisk $(*)$ in (B) and (G) indicates the depletion of CRBN in the tissue of the Crbn-KO mouse. Error bars indicate SEM. Statistical significance of differences was assessed using two-tailed Student's t-test. 
CRBN, in a combinatorial manner. In fact, there are numerous biochemical and structural properties of DCAFs that are tailored for the versatile CUL4-DDB1 core ubiquitin ligase (24).

A protein docking model of the CUL4-DDB1-CRBN complex derived from previously reported X-ray crystal structures (2527) is shown in Fig. 4. Other substrate receptors, such as Cockayne syndrome group A (CSA) and WD repeat-containing protein 5 (WDR5), were also shown for comparison. The PDB ID of each protein is reported in the figure legend. Over 50 DCAFs and their binding moiety with DDB1 have been reported with their expected functions (24). For example, CSA interacts with WD40 repeats that were originally characterized to engage in nucleotide excision repair along with DDB1 but are now recognized to also function as substrate receptors of the CUL4-DDB1 ubiquitin ligase (26). In addition to CRBN, each DCAF can recruit various cellular targets for their own functions as a substrate receptor. For this reason, we should consider the knockout-effect of each component in CRLs more carefully.

Considerable efforts have been made to identify new target proteins of CRBN and their roles in the normal and pathophysiology of the cell. Clinically, CRL4 ${ }^{\text {Crbn }}$ have been recognized as an important therapeutic target for several diseases, including multiple myeloma, leprosy, and hematopoietic cancer (28-30). CRBN is likely to affect a variety of brain-related functions and diseases; however, the physiological function of CRBN in the brain is still unclear. Thus, it is critical to under- stand the effects of CRBN modulation in terms of brain electrical activity and subsequent functional effects. The differential susceptibility to PTZ in Crbn-KO mice could provide a basis for a prudential approach to targeting CRL4 ${ }^{\mathrm{Crbn}}$. In order to ensure that the deletion of CRBN directly affects seizures induced by PTZ, we first need to select a group of protein candidates that are confirmed to have involvement in epileptic pathogenesis. It should then be verified that these candidate proteins conform to the control and regulation mechanism by CRL4 ${ }^{\text {Crbn }}$ using electrophysiological and molecular biological experiments. Thus, our findings showing that $\mathrm{Crbn}-\mathrm{KO}$ mice are not differentially susceptible to PTZ-induced seizure may provide a valuable example that the functional roles of E3 ligases should be interrogated at their individual substrates and substrate receptors, not the core components of the enzyme.

\section{MATERIALS AND METHODS}

\section{Westem blottingg}

For protein preparation, mice were terminally anesthetized using ketamine, and blood was removed from the organs by perfusion. The mouse tissues were lysed with a 1:10 ratio of Tris-Cl buffer (20 mM Tris-Cl [pH 7.4], $0.32 \mathrm{M}$ sucrose, $1 \mathrm{mM}$ EDTA, $1 \mathrm{mM}$ EGTA) using a homogenizer (Thomas Scientific). Protein samples were boiled with $2 \mathrm{X}$ sample buffer $(24 \mathrm{mM}$ Tris-Cl [pH 6.8], 10\% Glycerol, 0.04\% Bromophenol blue, 0.8\% SDS) to produce samples for Western blot analysis. Proteins were separated in $6-10 \%$ SDS-PAGE gels at $80-90 \mathrm{~V}$ in the stacking

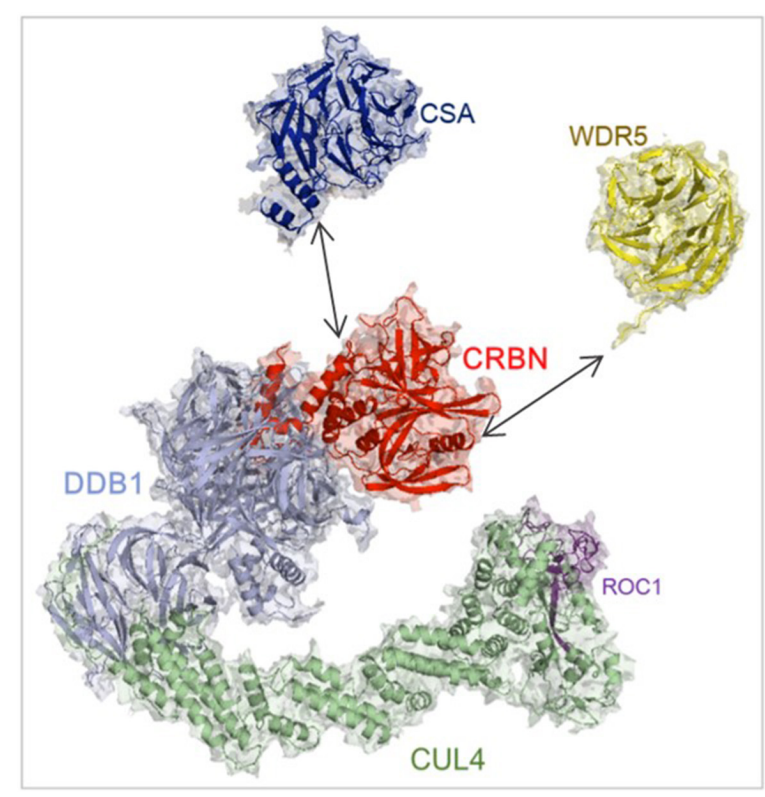

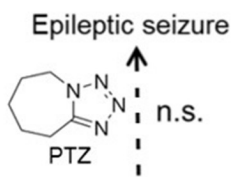

CRBN KO

Fig. 4. Structural modeling of CRL4-DDB1-CRBN with Cockayne syndrome group A (CSA) and WD40 repeat-containing protein 5 (WDR5). Protein modeling and structural interactions were determined using bioinformatics tools. The protein structure was determined by $\mathrm{X}$-ray crystallography and found in PDB ID CUL4: 2HYE, DDB1: 2HYE and 4CI1, CRBN: 4CI1, CSA: 6FCV, and WDR5: 4CY1. 
gel and $140-150 \mathrm{~V}$ in the running gel until the target protein reached the medial part of the gel. The proteins were subsequently transferred to a PVDF membrane (GE Healthcare Life Sciences, \#10600021). Membranes were blocked with 3\% BSA prepared in 1X TBS-T $(10 \mathrm{mM}$ Tris- $\mathrm{HCl}, 100 \mathrm{mM} \mathrm{NaCl}$, and $0.2 \%$ Tween 20, $\mathrm{pH}$ 7.5) for $50 \mathrm{~min}$. The blots were then incubated with the following primary antibodies: CRBN (HPA 045910), DDB1 (Ab9194), CUL4 (Ab71548), BK $\mathrm{Ca}_{\mathrm{a}}$ channel alpha (sc-374142), and TUBULIN (\#2144S). After washing twice in 1X TBS-T for $15 \mathrm{~min}$, the membranes were incubated with peroxidase-conjugated anti-rabbit IgG (Jackson ImmunoResearch Laboratories, \#111-035-003) or anti-mouse IgG (Jackson Immuno Research Laboratories, \#115-035-003) for 50 min at room temperature. After washing twice in 1X TBS-T for $15 \mathrm{~min}$, proteins were developed using enhanced chemiluminescence detection reagent (GE Healthcare Life Sciences, \#RPN2209).

\section{Experimental animals}

C57BL6J WT mice and Crbn-KO mice (19) (8 weeks, male) were housed in a room with a standard chow diet and water ad libitum in specific pathogen-free conditions (IVC-system) with a 12-h light-dark cycle. The mice in both groups did not show abnormal behaviors such as seizures without the application of chemoconvulsant. All materials for maintenance of animals were provided by the Gwangju Institute of Science and Technology Animal Care and Use Committee. All animal experiments were conducted according to the institutional guidelines of the Gwangju Institute of Science and Technology (GIST). This study was approved by the Animal Care Committee of GIST.

\section{Electroencephalography (EEG) recording and data analysis} Mice were anesthetized with a mixture of ketamine hydrochloride and xylazine $(0.2 \mathrm{mg} / \mathrm{g}, \mathrm{IM})$ and fixed to a stereotaxic frame (RWD Life Science Co., Shenzhen, China) using ear bars and a tooth bar. Depth electrodes were implanted into the right motor cortex (AP: $+2.0 \mathrm{~mm}, \mathrm{ML}:+2.0 \mathrm{~mm}$, DV: - 1.5) and hippocampus (AP: $-2.8 \mathrm{~mm}, \mathrm{ML}:+3.0 \mathrm{~mm}$, DV: -3.5 ). For referencing and grounding, two microelectrodes were implanted into the frontal lobe and cerebellum. The depth electrodes were made of twisted stainless-steel wires (Leico Industries, New York, USA) and the microelectrodes were made of stainless-steel screws and silver wires (Leico Industries, New York, USA). After recovery (7-10 days), EEG signals were acquired for $15 \mathrm{~min}$ after injection of PTZ at a sampling rate of $1000 \mathrm{~Hz}$ using the Plexon system (Plexon Inc, Dallas, TX, USA). Data analysis was performed using Matlab. To remove artifact signals, band-pass filtering $(1 \mathrm{~Hz}-120 \mathrm{~Hz})$ and notch filtering $(60 \mathrm{~Hz}$ and $120 \mathrm{~Hz}$ ) were applied. From the filtered signals, ictal periods were selected to represent the EEG patterns of seizure activity.

\section{Pentylenetetrazole-induced seizure}

PTZ was injected intraperitoneal at several different concentra- tions (20-200 mpk [milligram per kilogram]) to figure out the appropriate dose that induces seizures. Mice were then moved to empty cages and their behavior recorded for $30 \mathrm{~min}$. Seizures were classified into four stages using a well-established seizure stage scoring method, as previously described (5-7). Stage 1 was characterized by the mice being in the resting position with their abdomen touching the bottom of the cage. Stage 2 was defined as partial clonus. Stage 3 was described as generalized clonus of all four limbs. Stage 4 was defined as maximal seizures involving death.

\section{Bioinformatic data analysis}

We used the open source database PDB (Protein Data Bank, https://www.rcsb.org/) and PYMOL program for Cul4-DDB1CRBN E3 ubiquitin ligase modeling. The modeling for protein docking was performed using HEX (hex.loria.fr).

\section{Statistical analysis}

Data were quantified using Image (31), and graphs were produced with Microsoft Office PowerPoint 2013 and Origin 9.1. Data are expressed as mean \pm SEM. Significant differences between groups were determined by two-tailed unpaired Student's $t$-test.

\section{ACKNOWLEDGEMENTS}

This work was supported by GIST Research Institute (GRI) ARI grant funded by the GIST in 2020. This work was also supported by the National Research Foundation of Korea (NRF) grant funded by the Korea government (MSIT) (NRF-2019R1A2 C2087565).

\section{CONFLICTS OF INTEREST}

The authors have no conflicting interests.

\section{REFERENCES}

1. Bozzi Y, Casarosa S and Caleo M (2012) Epilepsy as a neurodevelopmental disorder. Front Psychiatry 3, 19

2. Jobe PC and Laird Ii HE (1987) Neurotransmitters and epilepsy, Springer Science \& Business Media

3. Zhu Y, Zhang S, Feng Y, Xiao Q, Cheng J and Tao J (2018) The Yin and Yang of BK channels in epilepsy. CNS Neurol Disord Drug Targets 17, 272-279

4. Hansen SL, Sperling BB and Sanchez C (2004) Anticonvulsant and antiepileptogenic effects of GABAA receptor ligands in pentylenetetrazole-kindled mice. Prog Neuro psychopharmacol Biol Psychiatry 28, 105-113

5. Ferraro TN, Golden GT, Smith GG et al (1999) Mapping loci for pentylenetetrazol-induced seizure susceptibility in mice. J Neurosci 19, 6733-6739

6. Harai T, Inoue R, Fujita Y et al (2012) Decreased susceptibility to seizures induced by pentylenetetrazole in serine racemase knockout mice. Epilepsy Res 102, 180-187 
7. Liu J, Ye J, Zou X et al (2014) CRL4A(CRBN) E3 ubiquitin ligase restricts BK channel activity and prevents epileptogenesis. Nat Commun 5, 3924

8. Higgins JJ, Pucilowska J, Lombardi RQ and Rooney JP (2004) A mutation in a novel ATP-dependent Lon protease gene in a kindred with mild mental retardation. Neurology 63, 1927-1931

9. Ito $T$, Ando H, Suzuki $T$ et al (2010) Identification of a primary target of thalidomide teratogenicity. Science 327, $1345-1350$

10. Lopez-Girona A, Mendy D, Ito T et al (2012) Cereblon is a direct protein target for immunomodulatory and antiproliferative activities of lenalidomide and pomalidomide. Leukemia 26, 2326-2335

11. Lee KM, Jo S, Kim H, Lee J and Park CS (2011) Functional modulation of AMP-activated protein kinase by cereblon. Biochim Biophys Acta 1813, 448-455

12. Nguyen TV, Lee JE, Sweredoski MJ et al (2016) Glutamine triggers acetylation-dependent degradation of glutamine synthetase via the thalidomide receptor cereblon. Mol Cell 61, 809-820

13. Robinson SR (2000) Neuronal expression of glutamine synthetase in Alzheimer's disease indicates a profound impairment of metabolic interactions with astrocytes. Neurochem Int 36, 471-482

14. Mates JM, Campos-Sandoval JA, Santos-Jimenez JL and Marquez J (2019) Dysregulation of glutaminase and glutamine synthetase in cancer. Cancer Lett 467, 29-39

15. Hohberger B and Enz R (2009) Cereblon is expressed in the retina and binds to voltage-gated chloride channels. FEBS Lett 583, 633-637

16. Pedersen $T H$, Riisager $A$, de Paoli FV, Chen TY and Nielsen OB (2016) Role of physiological ClC-1 Cl- ion channel regulation for the excitability and function of working skeletal muscle. J Gen Physiol 147, 291-308

17. Kang JA, Park SH, Jeong SP et al (2016) Epigenetic regulation of Kcna3-encoding Kv1.3 potassium channel by cereblon contributes to regulation of $\mathrm{CD} 4+\mathrm{T}$-cell activation. Proc Natl Acad Sci U S A 113, 8771-8776

18. Jo S, Lee KH, Song S, Jung YK and Park CS (2005) Identification and functional characterization of cereblon as a binding protein for large-conductance calcium-activated potassium channel in rat brain. J Neurochem 94, $1212-1224$
19. Lee KM, Yang SJ, Kim YD et al (2013) Disruption of the cereblon gene enhances hepatic AMPK activity and prevents high-fat diet-induced obesity and insulin resistance in mice. Diabetes 62, 1855-1864

20. Choi TY, Lee SH, Kim YJ et al (2018) Cereblon maintains synaptic and cognitive function by regulating BK channel. J Neurosci 38, 3571-3583

21. Fischer ES, Bohm K, Lydeard JR et al (2014) Structure of the DDB1-CRBN E3 ubiquitin ligase in complex with thalidomide. Nature 512, 49-53

22. Wu C, Orozco C, Boyer J et al (2009) BioGPS: an extensible and customizable portal for querying and organizing gene annotation resources. Genome Biol 10, 1-8

23. Bulatov E and Ciulli A (2015) Targeting Cullin-RING E3 ubiquitin ligases for drug discovery: structure, assembly and small-molecule modulation. Biochem J 467, 365-386

24. Lee J and Zhou P (2007) DCAFs, the missing link of the CUL4-DDB1 ubiquitin ligase. Mol Cell 26, 775-780

25. Angers S, Li T, Yi X, MacCoss MJ, Moon RT and Zheng N (2006) Molecular architecture and assembly of the DDB1-CUL4A ubiquitin ligase machinery. Nature 443, 590-593

26. Pines A, Dijk M, Makowski $M$ et al (2018) TRiC controls transcription resumption after UV damage by regulating Cockayne syndrome protein A. Nat Commun 9, 1040

27. Dias J, Van Nguyen N, Georgiev P et al (2014) Structural analysis of the KANSL1/WDR5/KANSL2 complex reveals that WDR5 is required for efficient assembly and chromatin targeting of the NSL complex. Genes Dev 28, 929-942

28. Shi Q and Chen L (2017) Cereblon: a protein crucial to the multiple functions of immunomodulatory drugs as well as cell metabolism and disease generation. J Immunol Res 2017, 9130608

29. Lupas AN, Zhu H and Korycinski M (2015) The thalidomidebinding domain of cereblon defines the CULT domain family and is a new member of the beta-tent fold. PLoS Comput Biol 11, e1004023

30. Han Y, Rajadhyaksha AM, Mailloux AW et al (2013) Characterization of RING finger E3-ubiquitin ligase cereblon in hematopoietic regulation. Blood 122, 1207-1207

31. Schneider CA, Rasband WS and Eliceiri KW (2012) NIH Image to Image): 25 years of image analysis. Nat Methods 9, 671-675 